\title{
Assessment of Left Ventricle Myocardial Function in Hypertensive Patients Using Three-Dimensional Speckle-Tracking Echocardiography (3D-STE)
}

\author{
Shima Ibrahim Ali1*; ${ }^{*}$ Awadia Gareeballah ${ }^{1,2}$; Rihab Ali Yousif ${ }^{1}$; Ahmed Abdelrahim \\ Mohammed $^{1}$; Marwa Hussain Mohammed ${ }^{1}$; Raga Ahmed Abouraida ${ }^{1}$; Mingxing XIE ${ }^{3}$ \\ ${ }^{I}$ Faculty of Radiological Sciences and Medical Imaging, Alzaiem Alazhari University \\ Khartoum North, Sudan \\ ${ }^{2}$ Department of Diagnostic Radiologic Technology, Faculty of Applied Medical Sciences, \\ Taibah University, Al-Madinah Al-Munawara, Saudi Arabia \\ ${ }^{3}$ Department of Ultrasound, Union Hospital, Tongji Medical College, \\ Huazhong University of Science and Technology, Wuhan, China
}

\begin{abstract}
Background: Increased myocardial fibrosis in hypertension leads to abnormalities in left ventricular diastolic function. 3D-speckle-tracking imaging (3D-STI) is a primary imaging modality used to detect early changes in the left ventricle (LV). The aim of this study was to assess the left ventricular myocardial function in hypertensive patients using 3D-speckle tracking imaging (3D-STI).

Methods and Results: A case control, nonintervention, descriptive study was conducted in the Department of Ultrasound Diagnosis of Union Hospital of Tongji Medical College of Huazhong University of Science and Technology (Wuhan, Hubei, China). The study subjects included 64 patients with hypertension (HT) and, as control group, 44 normotensives. HT patients were divided into HT-I group (SBP of 130-139 mmHg or DBP of 80-89 mmHg, and HT-II group (SBP $>140 \mathrm{mmHg}$ or DBP $>90$ $\mathrm{mmHg}$ ). In this study, LV geometry and function were assessed using conventional 2D- and 3D-echocardiography in a total of 108 consecutive subjects. LV volumes, global and regional strains were measured using 3D-STI. LV ejection fraction (LVEF) was in normal range in three groups, but in general, it slightly decreased in HT-II patients, compared with control and HT-I groups $(62.5 \pm 2.1 \%, 68.0 \pm 2.2 \%$, and $67.5 \pm 1.3 \%$, respectively, $P=0.00)$. Global systolic strain demonstrated a significant decrease in GLS, GCS, and GRS in the HT-II group, compared with control and HT-I groups. All regional strain parameters (longitudinal, circumferential, and radial) significantly decreased in HT-II patients, compared with control and HT-I groups.

Conclusion: A significant deterioration of global LV systolic functions is found in hypertensive patients with well-preserved LVEF, especially in patients with hypertension stage II.(International Journal of Biomedicine. 2021;11(4):403-409.)
\end{abstract}

Key Words: hypertension $\bullet$ left ventricle $\bullet$ speckle tracking $\bullet$ global strain $\bullet$ regional strain

For citation: Ali ShI, Gareeballah A, Yousif RA, Mohammed AA, Mohammed MH, Abouraida RA, XIE M. Assessment of Left Ventricle Myocardial Function in Hypertensive Patients Using Three-Dimensional Speckle-Tracking Echocardiography (3D-STE). International Journal of Biomedicine. 2021;11(4):403-409. doi:10.21103/Article11(4)_OA1

\begin{abstract}
Abbreviations
3D-STE, three-dimensional speckle-tracking echocardiography; 3D-STI, three-dimensional speckle-tracking imaging; ASE, American Society of Echocardiography; BP, blood pressure; BSA, body surface area; DBP, diastolic BP; EDV, end-diastolic velocity; GLS, global longitudinal strain; GCS, global circumferential strain; GRS, global radial strain; HT, hypertension; IVSd, interventricular septal thickness; LAd, left atrial dimension; LV, left ventricle; LVEF, left ventricular ejection fraction; LVEDV, left ventricular end-diastolic volume; LVESV, left ventricular end-systolic volume; LDEDd, left ventricular end-diastolic dimension; LVH, left ventricular hypertrophy; LVM, left ventricular mass; LVMI, LVM index; LVSV, left ventricular stroke volume; PWT, posterior wall thickness; RWT, relative wall thickness; SBP, systolic BP; SR, strain rate; SSR, systolic strain rate; SV, systolic velocity.
\end{abstract}




\section{Introduction}

According to the 2017 American College of Cardiology (ACC) and the American Heart Association (AHA) guideline, hypertension is defined as $\mathrm{SBP} \geq 130 \mathrm{mmHg}$ or $\mathrm{DBP} \geq 80$ mmHg. ${ }^{(1)}$ The prevalence of hypertension rises dramatically with increasing age and is higher in blacks than in whites, Asians, and Hispanic Americans. ${ }^{(1,2)}$

Early detection of LV dysfunction before the development of $\mathrm{LVH}$ is a finding that requires extensive treatment to reduce morbidity and mortality due to cardiovascular disease; therefore, it has to be considered in the evaluation of global cardiovascular risk. ${ }^{(3)}$

Echocardiography is still the primary imaging modality for assessing cardiac function; The most common index of the myocardial systolic function used in routine clinical practice is LVEF, but conversely, in several studies, LVEF was found to be suboptimal for detecting early myocardial dysfunction, likely due to undetectable wall deformation. . $^{(4,5)}$

3D-STE provides an accurate determination of subclinical myocardial dysfunction and also demonstrates comprehensive information about the cardiac ventricle. In addition, the accuracy and reproducibility of 3D-STE in evaluating the function and structure of the LV has been previously demonstrated. ${ }^{(6,7)}$

We hypothesize that 3D-STI may provide a reproducible measurement for the evaluation of LV subclinical myocardial changes in patients with primary hypertension.

\section{Materials and Methods}

A case control, nonintervention, descriptive study was conducted in the Department of Ultrasound Diagnosis of Union Hospital of Tongji Medical College of Huazhong University of Science and Technology (Wuhan, Hubei, China) during a period from June 2017 to May 2018. In this study, LV geometry and function were assessed using conventional 2D- and 3D-echocardiography in a total of 108 consecutive subjects. The study subjects included 64 HT patients and, as control group, 44 normotensives. HT patients were divided into HT-I group (SBP of $130-139 \mathrm{mmHg}$ or DBP of $80-89$ $\mathrm{mmHg}$, and HT-II group (SBP $>140 \mathrm{mmHg}$ or DBP $>90$ $\mathrm{mmHg}$ ).

\section{Inclusion criteria}

Patients aged $\geq 27$ years of different genders with a diagnosis of primary hypertension were included in hypertensive groups. The characteristic features of hypertension patients matched the diagnostic standards of the 2017 ACC/AHA guidelines. ${ }^{(8)}$

The control group included the participants with no hypertension or diabetes, no use of medication, no cardiac symptoms, and no abnormality detected in the heart in physical examination, electrocardiogram, and echocardiography results.

\section{Exclusion criteria}

Any patients with secondary causes of HT, coronary artery diseases, congenital heart disease, severe valvular stenosis or regurgitation, abnormal wall motion, heart failure, arrhythmia, pericardial effusion (moderate or massive) and lung disease were excluded.

\section{Echocardiography image acquisition}

The cardiac structure, chamber size, wall thickness, and cardiac function were evaluated by a conventional transthoracic echocardiogram, following the recommendations of the ASE. (9) A commercially available system (IE Elite, Philips Medical Systems, Andover, MA) with an S5-1 broadband phased-array transducer was used. A 3D echocardiographic examination was given to all subjects, using the same system and X5-1 transducer. Images were adjusted to assess the complete LV during a full-volume dataset within the four-chamber apical view. Datasets were acquired with an acquisition setting of four or seven heartbeats. End-expiratory breath holding was done whenever possible. At least four datasets were acquired for each subject, then for offline analysis, three datasets with the best image quality were selected. ${ }^{(10)}$ Datasets that did not include the whole LV, had indefinite endocardial borders, or had obvious stitch artifacts were excluded.

\section{Echocardiographic parameters}

The cardiac chamber size, LVM, and LVEF were measured according to the ASE chamber quantification guidelines..$^{(9)}$ IVSd, LVEDd, PWT, and LAd were measured in the parasternal long-axis view. RWT was calculated by dividing myocardial thickness (IVST+PWT) by LVEDd. Trans-mitral peak velocity of early diastole (E) and late diastole (A) was measured by pulse wave Doppler. The ratio of E/A was calculated. For the semi-automatic analysis of LVM, QLAB 3D (Philips Medical Systems) quantification software algorithms were used.

\section{D speckle-tracking echocardiography (3D-STE)}

Offline 3D-STE analysis was obtained with 4D LVAnalysis 3.0 (TomTec Imaging Systems, Unterschleissheim, Germany echocardiographic quantification software). The technique used for measurement is same as Zhang et al.: "Measurements were made using the data set with the best image quality, which was selected by consensus of the two readers. The frame rate of the volumetric image was 15 to 24 frames/sec. The 3D data sets were displayed as multiplanar reconstruction images corresponding to four tiles containing three standard LAX views and a short-axis view, which is orthogonal to the LAX views." (11) The offline analysis method was used. The following strain parameters were assessed during LV strain analysis: GLS (LV peak systolic global longitudinal strain), GRS (LV peak systolic global radial strain), GCS (LV peak systolic global circumferential strain). The three parameters were averaged over the 16 segments: "The peak value of each index was defined as its maximum absolute value with a positive or negative sign. Negative strain values reflect shortening, whereas positive strain values represent lengthening or thickening. All strain values are dimensionless and are expressed as percentages." ${ }^{\prime(11,12)}$

Statistical analysis was performed using the IBM SPSS Statistics V22.0 (SPSS Inc., Chicago, IL, USA). The 
normality of distribution of continuous variables was tested by one-sample Kolmogorov-Smirnov test. Continuous variables with normal distribution were presented as mean \pm standard deviation (SD). Multiple comparisons were performed with one-way ANOVA. Correlation coefficients $\left(\mathrm{R}^{2}\right)$ were calculated by linear regression analysis. A probability value of $P<0.05$ was considered statistically significant.

\section{Results}

\section{Clinical characteristics}

The demographic characteristics of two hypertensive groups (HT-I, HT-II) and the control group are summarized in Table 1. No significant differences in sex, height, and body surface area between the control, HT-I and HT-II groups were found.

\section{Table 1.}

Comparison between the study groups regarding demographic and clinical data

\begin{tabular}{|l|c|c|c|c|}
\hline \multicolumn{1}{|c|}{ Variables } & $\begin{array}{c}\text { Control group } \\
(\mathrm{n}=44)\end{array}$ & $\begin{array}{c}\text { HT-I group } \\
(\mathrm{n}=20)\end{array}$ & $\begin{array}{c}\text { HT-II group } \\
(\mathrm{n}=44)\end{array}$ & $P$-value \\
\hline Age, yrs & $53.5 \pm 7.9$ & $47.8 \pm 10.5^{*}$ & $56.3 \pm 11.6^{\#}$ & 0.01 \\
\hline Gender M/F & $30 / 14$ & $12 / 8$ & $30 / 14$ & 0.79 \\
\hline SBP, mmHg & $119.3 \pm 8.4$ & $135.7 \pm 3.9^{*}$ & $153.5 \pm 24.7^{* \#}$ & 0.00 \\
\hline DBP, mmHg & $75.2 \pm 9.8$ & $85.8 \pm 4.3^{*}$ & $93.5 \pm 19.1^{*}$ & 0.00 \\
\hline Height, cm & $1.6 \pm 0.8$ & $1.6 \pm 0.7$ & $1.6 \pm 0.1$ & 0.11 \\
\hline Weight, kg & $62.7 \pm 10.4$ & $67 \pm 9.6$ & $69.9 \pm 9.4^{*}$ & 0.00 \\
\hline BSA, m ${ }^{2}$ & $1.6 \pm 0.1$ & $1.7 \pm 0.1$ & $1.7 \pm 0.1$ & 0.10 \\
\hline
\end{tabular}

* Compared with control group, $P<0.05$; \# compared with HT-I group, $P<0.05$.

\section{D-echocardiographic analysis}

The study found significant differences in RWT between HT groups and normotensive controls $(0.4 \pm 0.1 \mathrm{~cm}$ for HT- I group and HT-II respectively, $0.3 \pm 0.1 \mathrm{~cm}$ for the control group, $P=0.03$ ). The parameters of diastolic function, such as $\mathrm{E} / \mathrm{A}$ ratio, show significant differences between the control, HT-I and HT-II groups $(1.4 \pm 0.9,0.9 \pm 0.3$, and $0.8 \pm 0.3$, respectively, $P=0.00)$. The LVEDd, PWT, LAd and LVM in the HT-II group were significantly higher than those of the control and HT-I groups (Table 2).

\section{D-STE analysis}

The 3D-echocardiography found no significant differences between the control, HT-I and HT-II groups, regarding end-diastolic velocity and systolic velocity (Table 3 ). A significant difference was found in LVESV among groups: it was higher in the HT-II group than in the control and HT-I groups $(42.8 \pm 10.7 \mathrm{~mL}, 35.7 \pm 6.7 \mathrm{~mL}$, and $37.2 \pm 8.4 \mathrm{~mL}$, respectively, $P=0.00)$. Concerning $L V E F$, the study revealed that it was in normal range in three groups, but in general, it slightly decreased in HT-II patients, compared with control and HT-I groups $(62.5 \pm 2.1 \%, 68.0 \pm 2.2 \%$, and $67.5 \pm 1.3 \%$, respectively, $P=0.00$ ). Global systolic strain demonstrated a significant decrease in GLS, GCS, and GRS in the HT-II group, compared with control and HT-I groups $(-15.9 \pm 1.6 \%,-23.5 \pm 1.4 \%$, and $-20.2 \pm 0.9 \%$, respectively, $P=0.00 ;-33.7 \pm 1.8 \%,-37.4 \pm 1.7 \%$, and $-38.6 \pm 1.3 \%$, respectively, $P=0.00 ;$ and $41.0 \pm 2.1 \%$, $51.1 \pm 1.7 \%$, and $49.0 \pm 1.4 \%$, respectively, $P=0.00)$. Compared with controls, GLS and GRS decreased $(P<0.05)$, but GCS in the HT-I group increased $(P<0.05)$ (Table 3$)$.

\section{Table 2.}

Comparison between the study groups by 2D-conventional echocardiography

\begin{tabular}{|l|c|c|c|c|}
\hline Variables & $\begin{array}{c}\text { Control group } \\
(\mathrm{n}=44)\end{array}$ & $\begin{array}{c}\text { HT-I group } \\
(\mathrm{n}=20)\end{array}$ & $\begin{array}{c}\text { HT-II group } \\
(\mathrm{n}=44)\end{array}$ & $P$-value \\
\hline LAd, cm & $2.9 \pm 0.3$ & $3.5 \pm 0.4^{*}$ & $3.9 \pm 0.4^{* \#}$ & 0.00 \\
\hline LVEDd, cm & $4.5 \pm 0.3$ & $4.6 \pm 0.4$ & $4.8 \pm 0.3^{* \#}$ & 0.00 \\
\hline IVSd, cm & $0.9 \pm 0.1$ & $1.0 \pm 0.1^{*}$ & $1.1 \pm 0.2^{* \#}$ & 0.00 \\
\hline PWT, cm & $0.8 \pm 0.1$ & $0.9 \pm 0.2^{*}$ & $1.0 \pm 0.2^{*}$ & 0.00 \\
\hline RWT, cm & $0.3 \pm 0.1$ & $0.4 \pm 0.1^{*}$ & $0.4 \pm 0.1^{*}$ & 0.03 \\
\hline LVM, g & $118.5 \pm 23.9$ & $146.0 \pm 39.0^{*}$ & $202.7 \pm 42.8^{* \#}$ & 0.00 \\
\hline E/A & $1.4 \pm 0.9$ & $0.9 \pm 0.3^{*}$ & $0.8 \pm 0.3^{*}$ & 0.00 \\
\hline
\end{tabular}

$L A d$, left atrium diameter; LVEDd, left ventricular end-diastolic dimension; IVSd, interventricular septum thickness; PWT, posterior wall thickness; RWT, relative wall thickness; LVM, left ventricular mass; * compared with control group, $P<0.05$; \# compared with HT-I group, $P<0.05$.

Table 3.

Comparison between the study groups by 3D-STE global strain parameters

\begin{tabular}{|l|c|c|c|c|}
\hline \multicolumn{1}{|c|}{ Variables } & $\begin{array}{c}\text { Control group } \\
(\mathrm{n}=44)\end{array}$ & $\begin{array}{c}\text { HT-I group } \\
(\mathrm{n}=20)\end{array}$ & $\begin{array}{c}\text { HT-II group } \\
(\mathrm{n}=44)\end{array}$ & $P$-value \\
\hline LVEDV, mL & $111.3 \pm 18.3$ & $113.8 \pm 26.6$ & $113.7 \pm 25.4$ & 0.87 \\
\hline LVESV, mL & $35.7 \pm 6.7$ & $37.2 \pm 8.4$ & $42.8 \pm 10.7^{* \#}$ & 0.00 \\
\hline LVSV, mL & $75.6 \pm 12.2$ & $76.6 \pm 18.3$ & $70.9 \pm 15.0$ & 0.21 \\
\hline LVEF, \% & $68.0 \pm 2.2$ & $67.5 \pm 1.3$ & $62.5 \pm 2.1^{* \#}$ & 0.00 \\
\hline GCS, \% & $-37.4 \pm 1.7$ & $-38.6 \pm 1.3^{*}$ & $-33.7 \pm 1.8^{* \#}$ & 0.00 \\
\hline GLS, \% & $-23.5 \pm 1.4$ & $-20.2 \pm 0.9^{*}$ & $-15.9 \pm 1.6^{* \#}$ & 0.00 \\
\hline GRS, \% & $51.1 \pm 1.7$ & $49.0 \pm 1.4^{*}$ & $41.0 \pm 2.1^{* \#}$ & 0.00 \\
\hline
\end{tabular}

$E D V$, end-diastolic volume; ESV, end-systolic volume; SV, stroke volume; $L V E F$, left ventricular ejection fraction; GLS, global longitudinal strain; GCS, global circumferential strain; GRS, global radial strain. ${ }^{*}$ compared with control group, $P<0.05$; ${ }^{*}$ compared with HT-I group, $P<0.05$. 
In 3D-STE of regional strain parameters, significant differences were demonstrated in this study in the longitudinal, circumferential, and radial (basal, middle and apical strains) strains among three groups. All regional strain parameters (longitudinal, circumferential, and radial) significantly decreased in HT-II patients, compared with control and HT-I groups (Table 4, Fig.1-3).

\section{Table 4.}

Comparison between the study groups by 3D-STE regional strain parameters

\begin{tabular}{|l|c|c|c|c|}
\hline Variables & $\begin{array}{c}\text { Control group } \\
(\mathrm{n}=44)\end{array}$ & $\begin{array}{c}\text { HT-I group } \\
(\mathrm{n}=20)\end{array}$ & $\begin{array}{c}\text { HT-II group } \\
(\mathrm{n}=44)\end{array}$ & $P$-value \\
\hline $\mathrm{CS} / \mathrm{B}, \%$ & $-31.8 \pm 3.6$ & $-32.2 \pm 3.1$ & $-29.5 \pm 3.1^{* \#}$ & 0.01 \\
\hline $\mathrm{CS} / \mathrm{M}, \%$ & $-39.9 \pm 3.2$ & $-41.5 \pm 2.1$ & $-37.2 \pm 2.8^{* \#}$ & 0.00 \\
\hline $\mathrm{CS} / \mathrm{A}, \%$ & $-45.1 \pm 3.5$ & $-47.1 \pm 4.0^{*}$ & $-39.1 \pm 4.7^{* \#}$ & 0.00 \\
\hline $\mathrm{RS} / \mathrm{B}, \%$ & $45.2 \pm 4.1$ & $41.6 \pm 2.8^{*}$ & $38.5 \pm 3.1^{* \#}$ & 0.00 \\
\hline $\mathrm{RS} / \mathrm{M}, \%$ & $50.9 \pm 4.5$ & $53.1 \pm 5.2$ & $41.8 \pm 3.3^{* \#}$ & 0.00 \\
\hline RSA,\% & $61.6 \pm 5.6$ & $59.0 \pm 4.0^{*}$ & $47.0 \pm 5.5^{* \#}$ & 0.00 \\
\hline LS/B,\% & $-23.2 \pm 3.4$ & $-18.0 \pm 2.0^{*}$ & $-17.1 \pm 3.0^{*}$ & 0.00 \\
\hline LS/M,\% & $-21.1 \pm 3.3$ & $-19.8 \pm 2.4$ & $-13.9 \pm 2.7^{* \#}$ & 0.00 \\
\hline LS/A,\% & $-27.9 \pm 4.7$ & $-24.6 \pm 2.6^{*}$ & $-17.7 \pm 3.5^{* \#}$ & 0.00 \\
\hline
\end{tabular}

$C S / B$, basal circumferential strain; $C S / M$, middle circumferential strain; $C S / A$, apical circumferential strain; $R S / B$, basal radial strain; $R S / M$, middle radial strain; $R S / A$ apical radial strain; $L S / B$, basal longitudinal strain; $L / M$, middle longitudinal strain; $L S / A$, apical longitudinal strain. ${ }^{*}$ Compared with control group, $P<0.01$; $\#$ compared with HT-I group, $P<0.01$.

Regarding the HT-I group, compared with control group, the basal radial strain (RS/B), apical radial stain (RS/A), basal longitudinal strain (LS/B), and apical longitudinal strain (LS/A) decreased while the apical circumferential strain (CS/A) increased. Among the patients of the HT-II group, a significant negative linear relationship was found between LVEF and global strain parameters [LVEF and GLS $\left(\mathrm{R}^{2}=0.12, P<0.05\right)$, LVEF and GCS $\left(\mathrm{R}^{2}=0.34, P<0.05\right)$, and LVEF and GRS $\left(\mathrm{R}^{2}=0.27, P<0.05\right)$, GCS was more relevant to LVEF (Fig.4).

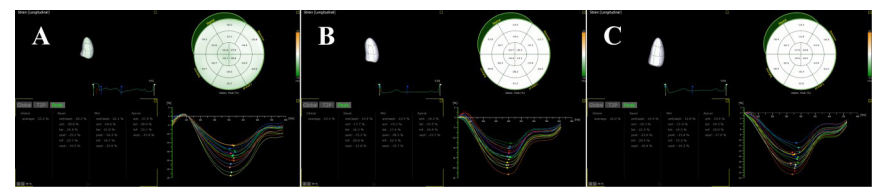

Fig.1. The LV longitudinal strain of HT-I and HT-II patients was lower than that of normotensive individual. The LV longitudinal strain of HT-II patient was the lowest. A normotensive individual; $B$ - HT-I patient; $C$ - HT-II patient. (These patients were all male patients of similar age).

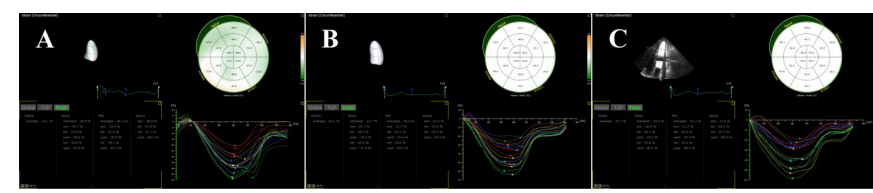

Fig.2. The LV circumferential strain of HT-II patient was lower than that of normotensive individual and HT-I patient. The LV circumferential strain of normotensive individual and HT-I patient was similar. $A$ - normotensive individual; $B$ - HT-I patient; $C$ - HT-II patient. (The same patients as in Figure 1).

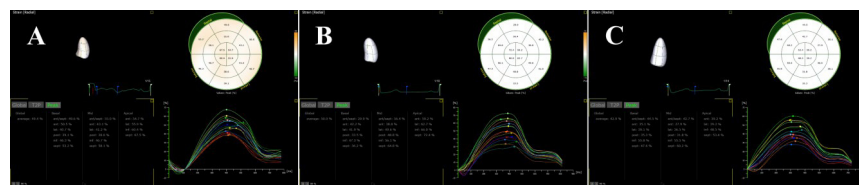

Fig.3. The LV radial strain of HT-II patient was lower than that of normotensive individual and HT-I patient. The LV radial strain of normotensive individual and HT-I patient was similar. A - normotensive individual; $B$ - HT-I patient; $C$ - HT-II patient. (The same patients as in Figures 1 and 2).

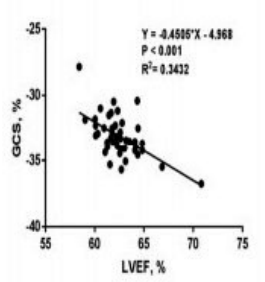

A

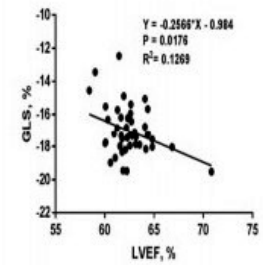

B

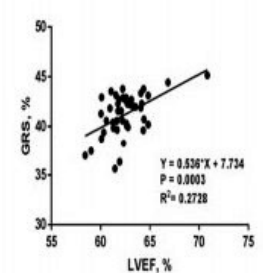

C
Fig.4. Relationship of LVEF with global systolic function in patients of HT-II group. A - GCS; B -GLS; C-GRS.

\section{Discussion}

The heart is a vital organ that sustains human life. In the late stage, systemic hypertension is one of the most frequent causes of heart failure.(5) Hypertension now accounts for the largest proportion of cardiovascular mortality worldwide. Cardiac hypertrophy occurs in hypertensive patients due to hemodynamic pressure overload, leading to increased wall thickness and LV remodeling. Echocardiography is the primary preferred imaging technique for assessing LV geometry and cardiac function for risk stratification. ${ }^{(13,14)}$

In this study, LV structure, mass, and E/A ratio were obtained from trans-mitral inflow Doppler. LV volumes, global and regional strains in longitudinal, circumferential, and radial directions were measured in primary diagnosed HT patients, using RT 3D-STE.

In 2D conventional echocardiography, in this study, the RWT was significantly increased in the hypertensive patients, compared to normotensive individuals; diastolic function parameters such as E/A ratio were significantly 
different between the control group, HT-I, and HT-II groups (decreased gradually from the control group to HT-1 and HT-II), but LVEDd, PWT, LAd and LVM were significantly higher in the HT-II than in the HT-1 and control groups. Compared to the present study, Ikonomidis et al. ${ }^{(15)}$ found that in $2 \mathrm{D}$ conventional echocardiography there was a non-significant difference in ejection fraction, LVEDV and LVESV between hypertensive and control groups; however, a significant difference was shown in LVMI, RWT, and the left atrial volume between the two groups; all these parameters were higher in a hypertensive group than in control group. Galderisi M. et al. ${ }^{(16)}$ also demonstrate the same results: Hypertensive patients have higher LVM and wall thickness and a significant difference in diastolic parameters, compared to control, but no significant difference in ejection fraction in conventional 2D echocardiography. No significant differences were found in this study in 3D-echocardiography between control, HT-I, and HT-II groups regarding EDV and SV. LVESV was slightly more in the HT-II group than in the control and HT-I groups. LVEF significantly decreased in HT-II, compared to HT-I and control groups, but was in the normal range. Furthermore, GCS, GLS, GRS showed significant differences between HT groups and control (significantly decreased in HT-II compared to HT- I and control groups).

Longitudinal basal, middle strain, apical strain, circumferential basal, middle and apical strain, radial basal, middle, and apical strain were decreased in patients of the HT-II group, compared to control and HT-I groups. Regarding the HT-I group, compared with control, CS/A increased, RS/B, RS/A, LS/B, and LS/A decreased. Among the patients of the HT-II group, a significant negative correlation was found between EF and each parameter of GLS, and GCS, with GCS being more relevant to LVEF. According to Galderisi et al., ${ }^{(16)}$ there was no significant difference in LV volumes, EF, or sphericity index determined by RT 3DE between the hypertensive and control groups, but LVMI was more in the hypertensive groups than in the control group. GAS and GLS were significantly decreased in hypertensive patients, but GCS did not vary significantly between hypertensive and control patients; in general, our results were close to theirs, except for GCS, as this study shows a significant difference in the control, HT-I and HT-II groups. $^{(16)}$

The results of this study are also similar to those of Yao et al.; ${ }^{(17)}$ they found that GAS, GLS, GCS, and GRS in hypertensive patients were significantly decreased, as was LVEF, compared to controls. Our study clarified that GAS can detect early changes in LV global systolic function in hypertensive patients with normal ventricular geometry; in our study, GCS is more relevant to LVEF. Haque et al. found that the global left ventricular, longitudinal, systolic strain, radial strain and area strain by RT3DSTE were significantly reduced $(P<0.001)$ in groups of hypertensive patients with presence or absence of LVH by $2 \mathrm{D}$-echo-cardiography, compared to the control group. ${ }^{(18)}$

In contrast to other studies conducted using 2D-STE to assess global and regional strains in hypertensive patients, El-Noamany et al. ${ }^{(19)}$ found that global peak systolic strain was significantly lower in the hypertensive group, compared to the control group. Global early diastolic SR was reduced in hypertensive patients, compared to controls $(P=0.001)$. Meanwhile, global late diastolic SR was higher in hypertensives than in the control group $(P=0.001)$. No significant differences in LVEF values and LV systolic function assessed by conventional echocardiography between hypertensives and control patients were noted. However, highly significant reductions in LV systolic strain and SR values were noted between control and hypertensive patients. ${ }^{(19)}$ Hamed et al. ${ }^{(20)}$ stated that the early diastolic SR was significantly decreased in hypertensive groups, compared with the control group, possibly due to increased myocardial fibrosis in hypertensive patients, leading to abnormalities in diastolic function and myocardial stiffness. GLS (global longitudinal strain) and SSR (systolic strain rate) were impaired in hypertensive patients, but the radial and circumferential strains showed insignificant differences between the control and hypertensive groups in a study done by Ikonomidis et al. ${ }^{(15)}$ This is because the longitudinal subendocardial fibers of the myocardium are most vulnerable to adverse influences, such as hypo-perfusion and agerelated interstitial fibrosis, so impaired longitudinal function, which is decreased in hypertensive disease, is an early sign of myocardial dysfunction. ${ }^{(21)}$

\section{Conclusion}

This study concludes that 3D-STE allows early diagnosis of abnormal LV myocardial systolic function, even in the subclinical stage. There was a significant reduction of myocardial systolic function in HT-II patients, compared with HT-I patients and the control group. The basal, middle, and apical segments strains significantly decreased in hypertensive patients, compared to the control group. GCS (global circumferential strain) may play a more important role in maintaining LVEF (left ventricular ejection fraction) than GLS (global longitudinal strain) and GRS (global radial strain).

\section{Ethical Clearance}

The ethical clearance was obtained from the Department of Ultrasound Diagnosis of Union Hospital of Tongji Medical College of Huazhong University of Science and Technology, Wuhan, Hubei, China. All the study participants were informed that all information would be used for research purposes only, then the result of the examination formed a part of this study.

All specific information relating to the participants' identities was protected, as was other medical data collected as routine case management. Participants were informed about the procedure and purpose of the study. Additional informed consent was obtained from all individual participants for whom identifying information is included in this article. Ultrasound and echocardiography scanning formed a part of routine management of the study cases. 


\section{Acknowledgements}

The authors want to extend thanks to the Ultrasound Diagnosis Department of Union Hospital of Tongji Medical College of Huazhong University of Science and Technology (Wuhan, Hubei, China) for granted approval for data collection and assistance in data collection for this study.

\section{Competing Interests}

The authors declare that they have no competing interests.

\section{References}

1. Whelton PK, Carey RM, Aronow WS, Casey DE Jr, Collins KJ, Dennison Himmelfarb C, DePalma SM, Gidding S, Jamerson KA, Jones DW, MacLaughlin EJ, Muntner P, Ovbiagele B, Smith SC Jr, Spencer CC, Stafford RS, Taler SJ, Thomas RJ, Williams KA Sr, Williamson JD, Wright JT Jr. 2017 ACC/AHA/AAPA/ABC/ACPM/AGS/APhA/ASH/ ASPC/NMA/PCNA Guideline for the Prevention, Detection, Evaluation, and Management of High Blood Pressure in Adults: A Report of the American College of Cardiology/American Heart Association Task Force on Clinical Practice Guidelines. J Am Coll Cardiol. 2018 May 15;71(19):e127-e248. doi: 10.1016/j.jacc.2017.11.006.

2. Schwartzbard AZ, Newman JD, Weintraub HS, Baum SJ. The 2017 high blood pressure clinical practice guideline: The old and the new. Clin Cardiol. 2018 Mar;41(3):279-281. doi: $10.1002 /$ clc. 22905 .

3. Cuspidi C, Meani S, Valerio C, Fusi V, Sala C, Zanchetti A. Left ventricular hypertrophy and cardiovascular risk stratification: impact and cost-effectiveness of echocardiography in recently diagnosed essential hypertensives. J Hypertens. 2006 Aug;24(8):1671-7. doi: 10.1097/01.hjh.0000239305.01496.ca.

4. Yip GW, Zhang Q, Xie JM, Liang YJ, Liu YM, Yan B, Lam YY, Yu CM. Resting global and regional left ventricular contractility in patients with heart failure and normal ejection fraction: insights from speckle-tracking echocardiography. Heart. 2011 Feb;97(4):287-94. doi: 10.1136/hrt.2010.205815. Epub 2010 Dec 30. Erratum in: Heart. 2011 Mar;97(6):516. Xie, Jun-Mei [corrected to Xie, Jun-Min].

5. Lafitte S, Perlant M, Reant P, Serri K, Douard H, DeMaria A, Roudaut R. Impact of impaired myocardial deformations on exercise tolerance and prognosis in patients with asymptomatic aortic stenosis. Eur J Echocardiogr. 2009 May;10(3):414-9. doi: 10.1093/ejechocard/jen299.

6. Maffessanti F, Nesser HJ, Weinert L, SteringerMascherbauer R, Niel J, Gorissen W, Sugeng L, Lang RM, Mor-Avi V. Quantitative evaluation of regional left ventricular function using three-dimensional speckle tracking echocardiography in patients with and without heart disease. Am J Cardiol. 2009 Dec 15;104(12):1755-62. doi: 10.1016/j. amjcard.2009.07.060.

7. Nesser HJ, Mor-Avi V, Gorissen W, Weinert L, SteringerMascherbauer R, Niel J, Sugeng L, Lang RM. Quantification of left ventricular volumes using three-dimensional echocardiographic speckle tracking: comparison with MRI. Eur Heart J. 2009 Jul;30(13):1565-73. doi: 10.1093/eurheartj/ ehp187.

8. Whelton PK, Carey RM. The 2017 Clinical Practice Guideline for High Blood Pressure. JAMA. 2017 Dec 5;318(21):2073-2074. doi: 10.1001/jama.2017.18209.

9. Saltijeral A, Perez de Isla L, Veras K, Fernandez Mde J, Gorissen W, Rementeria J, Almeria C, Rodrigo JL, Fernandez-Golfin C, Marcos-Alberca P, Macaya C, Zamorano J. Myocardial strain characterization in different left ventricular adaptative responses to high blood pressure: a study based on 3D-wall motion tracking analysis. Echocardiography. 2010 Nov;27(10):1238-46. doi: 10.1111/j.1540-8175.2010.01234.X.

10. Liu HY, Deng YB, Liu K, Li Y, Tang QY, Wei X, Chang $\mathrm{S}, \mathrm{Lu} \mathrm{X}$. Left ventricular systolic strain of the cardiac allograft evaluated with three-dimensional speckle tracking echocardiography. J Huazhong Univ Sci Technolog Med Sci. 2013 Oct;33(5):765-769. doi: 10.1007/s11596-0131194-8.

11. Zhang L, Gao J, Xie M, Yin P, Liu W, Li Y, Klas B, Sun J, Balluz R, Ge S. Left ventricular three-dimensional global systolic strain by real-time three-dimensional speckletracking in children: feasibility, reproducibility, maturational changes, and normal ranges. J Am Soc Echocardiogr. 2013 Aug;26(8):853-9. doi: 10.1016/j.echo.2013.05.002.

12. Monte IP, Mangiafico S, Buccheri S, Arcidiacono AA, Lavanco V, Privitera F, Leggio S, Deste W, Tamburino C. Early changes of left ventricular geometry and deformational analysis in obese subjects without cardiovascular risk factors: a three-dimensional and speckle tracking echocardiographic study. Int J Cardiovasc Imaging. 2014 Aug;30(6):1037-47. doi: 10.1007/s10554-014-0429-5.

13. Rosei EA, de Simone G, Mureddu G, Trimarco B, Verdecchia P, Volpe M, et al. Arterial hypertension and cardiac damage: diagnostic and therapeutic guidelines. High Blood Pressure \& Cardiovascular Prevention; Auckland. 2008 July:15(3):141-70. doi: 10.2165/0151642-20081503000008

14. Sadler DB, Aurigemma GP, Williams DW, Reda DJ, Materson BJ, Gottdiener JS. Systolic function in hypertensive men with concentric remodeling. Hypertension. 1997 Oct;30(4):777-81. doi: 10.1161/01.hyp.30.4.777.

15. Ikonomidis I, Tzortzis S, Triantafyllidi H, Parissis J, Papadopoulos C, Venetsanou K, Trivilou P, Paraskevaidis I, Lekakis J. Association of impaired left ventricular twistinguntwisting with vascular dysfunction, neurohumoral activation and impaired exercise capacity in hypertensive heart disease. Eur J Heart Fail. 2015 Dec;17(12):1240-51. doi: 10.1002/ ejhf.403.

16. Galderisi M, Esposito R, Schiano-Lomoriello V, Santoro A, Ippolito R, Schiattarella P, Strazzullo P, de Simone G. Correlates of global area strain in native hypertensive patients: a three-dimensional speckle-tracking echocardiography study.

"Corresponding author: Shima Ibrahim Ali, Radiological Sciences and Medical Imaging, Alzaiem Alazhari University, Khartoum North, Sudan. E-mail: SHIMAIBRAHIM@aau.edu.sd 
Eur Heart J Cardiovasc Imaging. 2012 Sep;13(9):730-8. doi: 10.1093/ehjci/jes026.

17. Yao H, Li J, Lin J, Huang B, Huang H. [Assessment of global left ventricular function in hypertensive patients with normal ventricular geometry using global area strain]. Sheng Wu Yi Xue Gong Cheng Xue Za Zhi. 2013 Apr;30(2):306-11. [Article in Chinese].

18. Haque T, Siddique R, Kabiruzzaman M, Khan AM, Choudhury S, Malik F. A11256 Assessment of Subclinical Systolic Dysfunction in Hypertensive Patients with Preserved Left Ventricular Ejection Fraction Using Three-Dimensional Speckle Tracking Echocardiography. Journal of Hypertension. October 2018;36: e174. doi: 10.1097/01.hjh.0000548707. 42035.ee

19. El-Noamany MF, Dawood AAE, Hamed WAI, Amer NE.
Assessment of Left Ventricular Functions in Hypertensive Diabetic Patients by Speckle Tracking Imaging: Correlation with Brain Natriuretic Peptide Levels. Cardiology and Cardiovascular Research. January 2020;4(3):131. doi: 10.11648 /j.cc r. 20200403.19

20. Hamed WAI, Kamal AM, Noamany MF, Soliman MA, Ra'ouf MMA. Evaluation of left ventricular performance in hypertensive patients by speckle tracking echocardiography: Correlation with brain natriuretic peptide. The Egyptian Heart Journal. 2014;66(4):299-308. doi: 10.1016/j. ehj.2014.08.002

21. Lai YH, Lo CI, Wu YJ, Hung CL, Yeh HI. Cardiac Remodeling, Adaptations and Associated Myocardial Mechanics in Hypertensive Heart Diseases. Acta Cardiol Sin. 2013 Jan;29(1):64-70. 